\title{
Simulation of particle motion in a closed conduit validated against experimental data
}

\author{
Jindřich Dolanský1, a \\ ${ }^{1}$ Institute of Hydrodynamics, ASCR, Pod Păankou 5, Prague, Czech Republic
}

\begin{abstract}
Motion of a number of spherical particles in a closed conduit is examined by means of both simulation and experiment. The bed of the conduit is covered by stationary spherical particles of the size of the moving particles. The flow is driven by experimentally measured velocity profiles which are inputs of the simulation. Altering input velocity profiles generates various trajectory patterns. The lattice Boltzmann method (LBM) based simulation is developed to study mutual interactions of the flow and the particles. The simulation enables to model both the particle motion and the fluid flow. The entropic LBM is employed to deal with the flow characterized by the high Reynolds number. The entropic modification of the LBM along with the enhanced refinement of the lattice grid yield an increase in demands on computational resources. Due to the inherently parallel nature of the LBM it can be handled by employing the Parallel Computing Toolbox (MATLAB) and other transformations enabling usage of the CUDA GPU computing technology. The trajectories of the particles determined within the LBM simulation are validated against data gained from the experiments. The compatibility of the simulation results with the outputs of experimental measurements is evaluated. The accuracy of the applied approach is assessed and stability and efficiency of the simulation is also considered.
\end{abstract}

\section{Introduction}

Motion of multiple particles in the turbulent flow represents an important application of fluid dynamics. The detailed and accurate description of particle motion in the flow belongs to difficult problems with many inputs. The motion is determined by the mutual particle-particle, particle-wall and particle-flow interactions and depends on the flow parameters as velocity, solids concentration, density, shape, and size distribution of the conveyed solid objects, the diameter and roughness of the pipe. Understanding of underlying particle-fluid dynamics is important for the safe and economical design of the transport technology.

Transport of spherical particles in a closed conduit with a rough bed is considered as an example of such a process. The particles are conveyed by the water flow defined by the specific velocity profiles. The particles perform both translational and rotational motions, influence the flow around them and undergo collisions with themselves and with the boundaries of the conduit. The bed of the conduit is covered by stationary spherical particles of the size of the moving particles.

The considered system was examined experimentally on a pipe loop with a test section where several trajectories of individual particles were observed. The particles moved mostly in a layer close to the pipe invert, however with increasing velocity more particles moved in saltation mode, some of them even freely suspended in the central or upper portion, e.g. [1]

The mathematical model of the system consists of equations for the fluid flow (e.g., Navier-Stokes), equations of motion for particles (Newton equations) and equations for velocities before and after (both particle-bed and particle-

\footnotetext{
a e-mail: dolansky@ih.cas.cz
}

particle) collisions which can be derived from relations for impulse forces.

To solve the flow equations the lattice Boltzmann method (LBM) is used. The LBM is a two decade old numerical approach originating from the lattice gas automata methods (LGCA) used for simulations of complex fluid flows, e.g., [2-4]. The LBM represents a second order and efficient computational scheme - due to its inherent locality and explicitness which enable straightforward parallelization. Moreover, it was shown that the incompressible NavierStokes equations can be derived from the lattice Boltzmann equations through the Chapman-Enskog procedure in the limit of long wavelengths as compared to the lattice scale, e.g., [5].

The LBM based simulation is developed to study mutual interactions of the flow and the particles. Except it is employed as a flow solver the LBM also enable to compute hydrodynamic forces of the fluid acting on the macroscopic particles. The hydrodynamic forces enter the Newton equations which are every time step coupled to the lattice Boltzmann equations to determine motion of the particles in the flow. Thus the LBM provides a unique computational frame for solving the particle-fluid systems, e.g., [6].

However, the basic LBM is subject to instabilities for the processes described by the high Reynolds numbers. As the considered system is characterized by the Reynolds number up to $10^{5}$ the entropic extension of the LBM is employed. The entropic modification of the LBM and the enhanced refinement of the lattice grid yield an increase in demands on computational resources. It is handled by employing the Parallel Computing Toolbox (MATLAB) and other transformations enabling usage of the CUDA GPU computing technology. 


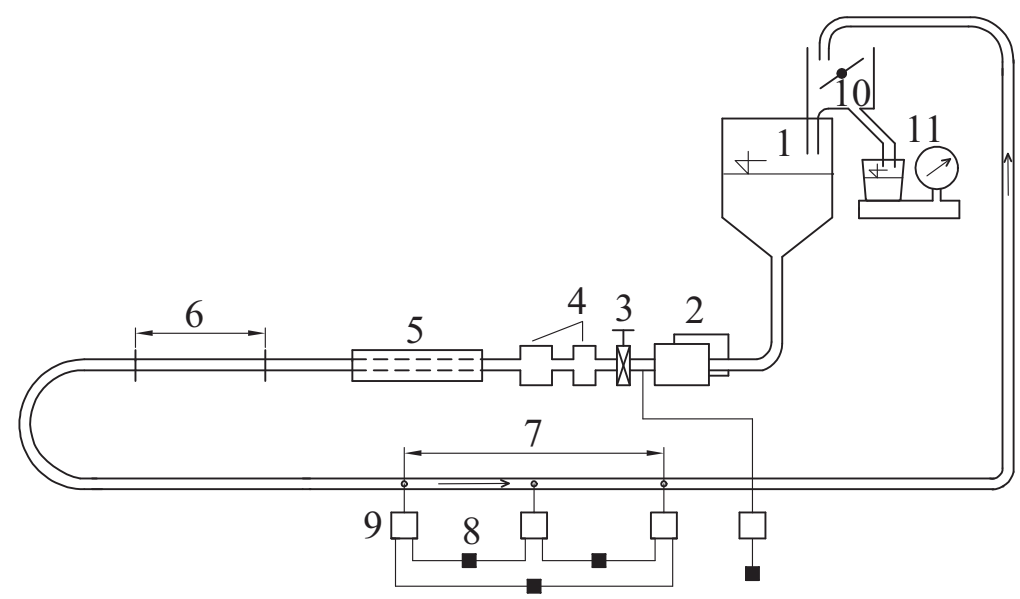

Fig. 1. Layout of the experimental pipeline loop (1-slurry tank, 2-pumps, 3-control valve, 4-flow meters, 5-heat exchanger, 6-transparent section, 7-measurement section, 8-pressure transducer, 9-sedimentation vessels, 10-flow divider, 11-density and discharge measurement).

The trajectories of the particles computed by the LBM simulation are validated against data gained from the experiments. The measured velocity profiles serve as inputs of the simulation and correspond to a number of flow rates. The different experimental velocity profiles generate various particle trajectory patterns.

The compatibility of the simulation results with the outputs of experimental measurements is evaluated. The accuracy of the applied approach is assessed and stability and efficiency of the simulation is also considered.

This paper proceeds as follows. In the next section, Sect. 2, the experimental tools and material is described. The mathematical model of the process is introduced in Sect. 3. In the following section, Sect. 4, the LBM approach is briefly summarized. The developed simulation is analyzed in Sect. 5. In Sect. 6 the results of the simulation and experiments are presented and discussed. Finally, the conclusions are inferred in Sect. 7.

\section{Measurements}

The considered system were measured on an experimental pipe loop with a test section of smooth stainless steel pipes with an inner diameter $36 \mathrm{~mm}$, cf. figure 1 . A two meter long transparent pipe viewing section (glass tube of inner diameter $D=40 \mathrm{~mm}$ equipped with special optical box), was used for a visual observation of particle and carrier liquid flow patterns, which were recorded using the digital camera, NanoSenze MKIII+, cf. [7].

The particle-water mixture was forced by a booster pump WARMAN 3/2 C - AH from an open storage tank, a variable speed drive was used to control mixture flow rates. The mixture flow rate and concentration were measured by a KROHNE-CORIMASS-800 G+ mass flow-meter. The temperature of the mixture was maintained by the heat exchanger.

The moving particles were supplied by glass balls of uniform size distribution (particle radius $r=3 \mathrm{~mm}$ and density $\rho=2540 \mathrm{~kg} \mathrm{~m}^{-3}$ ). A stationary bed layer was created in the viewing section of the pipe from particles of the same shape and size as the conveyed particles. The lead shots $\left(\rho=11340 \mathrm{~kg} \mathrm{~m}^{-3}\right)$ were used to satisfy requirement of stationarity of the bed layer with respect to the effect of the flow. The stationary bed layer was created from two layers of shots with its height varying from 9 to $12 \mathrm{~mm}$ from pipe invert, and resulting bed roughness was $k=3$ $\mathrm{mm}$.

The PIV (Particle Image Velocity) measurements were performed in a horizontal pipe section equipped with special optical box around the glass pipe to evaluate the local water velocity $u$. A continuous light sheet with a thickness of $1.5 \mathrm{~mm}$ was oriented in the stream-wise direction on the centerline plane. The aluminum powder with mean diameter of $10 \mu \mathrm{m}$ was used for tracking particles. The images were recorded by a high speed camera NanoSence MK III with image resolution $1280 \times 512$ pixels and frame rate 1510 $\mathrm{Hz}$.

The effect of stationary bed on the velocity profiles is illustrated in figure 2 for different flow rates $Q$. The measured velocity profiles were asymmetrical, maximum velocity values were at distance about $h=23 \mathrm{~mm}$ above the pipe invert. The velocity gradient was steeper in the lower part of the profile and local velocities near the stationary bed (from $h=9$ to $12 \mathrm{~mm}$ ) were practically equal zero due to the high value of the bed roughness.

\section{Mathematical model}

The flow field is described by the incompressible NavierStokes equations

$$
\rho_{f}\left(\frac{\partial \mathbf{u}}{\partial t}+\mathbf{u} \cdot \nabla \mathbf{u}\right)=-\nabla p+\mu \nabla^{2} \mathbf{u},
$$

with constant kinematic viscosity $v$. The fluid flow is driven by velocity profiles determined from experimental measurements which are illustrated in figure 2 . The various profiles are distinguished by the different flow rate $Q$.

Both the walls of the conduit and the surfaces of the moving particles are solid. The bed is semi-regularly covered by stationary solid spherical particles. Thus, the noslip boundary conditions (i.e., identification of the fluid velocity adjacent to the surface with the velocity of the surface) are defined for both cases as

$$
\mathbf{u}\left(\mathbf{x}_{b}\right)=0, \quad \text { respectively } \quad \mathbf{u}\left(\mathbf{x}_{b}\right)=\mathbf{v}\left(\mathbf{x}_{b}\right),
$$




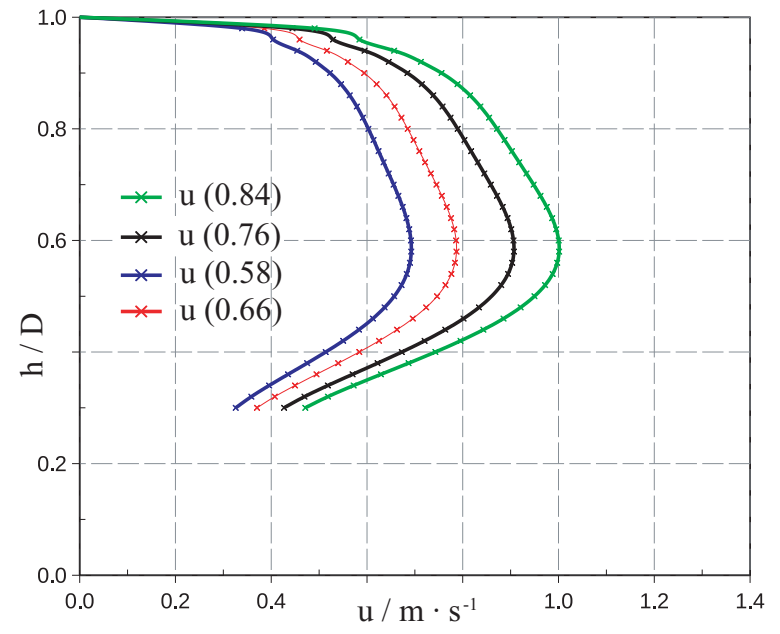

Fig. 2. The velocity profiles for different flow rates $Q$ are depicted in various colors: $Q=0.581 \mathrm{~s}^{-1}$ in blue, $Q=0.661 \mathrm{~s}^{-1}$ in red, $Q=0.761 \mathrm{~s}^{-1}$ in black and $Q=0.841 \mathrm{~s}^{-1}$ in green. The effect of stationary bed made of spherical particles is manifested in shapes of the profiles.

where $\mathbf{u}$ represents flow velocity and $\mathbf{v}$ stands for particle velocity in a boundary point $\mathbf{x}_{b}$.

Motion of solid particles in the flow is determined by actions of body and hydrodynamic forces. The body force is represented by the gravitational force $\mathbf{F}_{g}$. The hydrodynamic forces consists of the sum of the drag force $\mathbf{F}_{d}$, the force due to added mass $\mathbf{F}_{m}$ and the lift force $\mathbf{F}_{L}$, e.g., [8]. The resultant force also develops a torque on the particles which determines their angular velocities $\omega$.

Both the particle-bed and particle-particle collision models are derived from impulse equations of the form $m\left(\mathbf{v}^{\prime}-\right.$ $\mathbf{v})=\mathbf{J}$ which use the impulse force $\mathbf{J}$ as the measure of change of momentum (the quote mark distinguishes velocities before and after collisions). It is supposed that collisions take place in a very short time and all external forces can be neglected.

If rotation is taken into account the corresponding impulse equation for the angular velocities before and after the collision reads as $I\left(\omega^{\prime}-\omega\right)=r \mathbf{J}_{t}$ where $I$ stands for momentum of inertia and $\mathbf{J}_{t}$ is the tangential component of the impulse force. The above relations enable to derive expressions for new velocities after collisions for both the particle-bed and particle-particle collisions, cf. [9-11]).

\section{Numerical method}

The numerical model of the above described system is based on the LBM approach. In the LBM the fluid is composed of fictive particles which propagate along the lattice links and interact in nodes. A fictive particle is represented by the particle distribution function $f\left(\mathbf{x}, \mathbf{c}_{i}, t\right)$ which give probability of finding the fictive particle in a node $\mathbf{x}$ with a certain discrete velocity $\mathbf{c}_{i}$ in time $t$. Dynamics of fictive particles is described by the lattice Boltzmann equation

$$
f_{i}\left(\mathbf{x}+\mathbf{c}_{i} \Delta t, t+\Delta t\right)-f_{i}(\mathbf{x}, t)=\Gamma_{i},
$$

where the term $\Gamma_{i}$ on the right-hand side represents a collision operator which is required to fulfill the first law of thermodynamics, i.e., in this case conservation of mass and momentum ( $\Delta t$ is the lattice time step). The simple and frequent choice is represented by the Bhatnagar-Gross-Krook (BGK) collision operator

$$
\Gamma_{i}=\frac{1}{\tau}\left(f_{i}^{e q}-f_{i}\right)
$$

It expresses the tendency of the particle distributions $f_{i}$ to local equilibria $f_{i}^{e q}$ and the so called relaxation parameter $\tau$ expresses the rate of the tendency to the local equilibrium $f_{i}^{e q}$.

Macroscopic boundary conditions have to be expressed with the help of lattice numerical schemes which represent them on the microscopic level of the fictive particles. For the no-slip boundary condition the so called bounce-back scheme is employed

$$
f_{i}\left(\mathbf{x}_{b}, t+1\right)=f_{-i}\left(\mathbf{x}_{b}, t+1 / 2\right),
$$

which consists in inversions of particle distributions along the directions incident to the boundary nodes, denoted by exchange $i$ for $-i$. In the case of the moving surface the term corresponding to the exchange of momentum between fictive particles and the moving macroscopic particle has to be added to the inverted distributions

$$
f_{i}\left(\mathbf{x}_{b}, t+1\right)=f_{-i}\left(\mathbf{x}_{b}, t+1 / 2\right)+2 \frac{w_{i} \rho\left(\mathbf{x}_{b}, t\right)}{c_{s}^{2}}\left(\mathbf{c}_{i} \cdot \mathbf{v}\right),
$$

where $w_{i}$ represents weights of respective discrete velocities $\mathbf{c}_{i}, \rho$ is the local density and $c_{s}^{2}$ is the lattice sound speed, cf. [12]. Thus, the additional term represents the action of a macroscopic object on the fictive particles, i.e., on the flow.

The velocity profiles specified by experimental measurements are implemented by the Zou-He boundary scheme [13] as it allows to impose given velocity $\mathbf{u}\left(\mathbf{x}_{i n}, t\right)$ or pressure $p\left(\mathbf{x}_{i n}, t\right)$ on the boundary. In this case, only the nonequilibrium parts of distributions $f_{i}-f_{i}^{e q}$ are bounced-back in the normal direction with respect to the boundary.

In the LBM frame motion of macroscopic objects is effect of interaction of fictive particles with surfaces of these objects. More precisely, such a motion is caused by the momentum transfer $\Delta \mathbf{p}$ from the fictive particles to the macroscopic particles. Time rate of this momentum transfer $\Delta \mathbf{p} / \Delta t$ defines the hydrodynamic forces by which the flow acts on the objects. The hydrodynamic forces are then calculated as a sum over momentum contributions from all fictive particles incident to the boundary nodes of the objects $\mathbf{F}_{b}=-\Delta \mathbf{p} / \Delta t$ where

$$
\Delta \mathbf{p}=2 \mathbf{c}_{-i}\left(f_{i}\left(\mathbf{x}_{b}, t\right)-\frac{w_{i} \rho\left(\mathbf{x}_{b}, t\right)}{c_{s}^{2}}\left(\mathbf{c}_{i} \cdot \mathbf{v}\right)\right),
$$

cf. [14]. Another two contributions to the hydrodynamic forces comes from nodes covered or uncovered by motion of a particle. The impulse force $\mathbf{F}_{c o v}$ exerted on the particle is given by momentum sum over covered nodes from the previous time step while the force $\mathbf{F}_{\text {ucov }}$ gives the negative impulse force increment corresponding to momentum from uncovered nodes. The total hydrodynamic forces is then expressed as the sum $\mathbf{F}_{b}+\mathbf{F}_{c o v}+\mathbf{F}_{u n}$.

To update the particle position $\mathbf{x}(t)$ and its velocity $\mathbf{v}(t)$ the left-hand side of the Newton equations has to be integrated every time step. For this purpose, the leap-frog algorithm is chosen as it is simple, possesses second order 
accuracy, is invariant under time reversal and has other favorable global properties [15].

\section{Simulation}

In the last years a number of simulation tools based on the LBM have been developed. The various softwares differ in the application domains, in the ways of implementation, in employments of various programming resources and in overall robustness of their design. To assess capacity of the LBM based simulations in comparison with the traditional CFD approaches in aspects of efficiency, accuracy and stability validations against experiments or numerical methods are performed.

The stability of the LBM simulations is endangered for processes characterized by high Reynolds numbers in which case the relaxation parameter tends to the critical value $\tau \rightarrow 1 / 2$ equivalent to the inviscid flow. In this case the LBM becomes unstable because of incapability to dissipate the energy due to very large velocity gradients. The instability issues are eliminated by various approaches and extensions, e.g., the multi-relaxation time (MRT) method or the regularized LBM or the so called the entropic LBM.

In the developed simulation the last mentioned modification of the LBM is employed. Thus, if the parameter $\tau$ in the BGK approximation (1) is replaced by the factor $\alpha / 2 \tau$ where the parameter $\alpha$ represents a non-trivial root of equation

$$
H\left(f_{i}+\alpha\left(f_{i}^{e q}-f_{i}\right)\right)=H\left(f_{i}\right),
$$

with the Boltzmann $\mathrm{H}$-function of the form

$$
H(f)=\sum_{i} f_{i} \ln \frac{f_{i}}{w_{i}}
$$

then the second thermodynamic law is supported in the collision process, i.e., the entropy cannot decrease in the process. It results in unconditional stability of the method even for high Reynolds number cases - while still retaining its locality and efficiency (Karlin et al., 2002, 2006 [16, 17]).

However, calculation of the parameter $\alpha$ in each (potentially disruptive) node means solving the non-linear equation (2). This equation is usually solved by combination of the bisection method and the Newton method. The first method assumes that the interval inside which a solution exists is known while in the second method a sufficiently closed estimate to the solution should be known. Neither of them is trivial.

The upper estimate of the $\alpha_{\max }$ parameter can be easily found as the minimal solution to the equations

$$
f_{i}+\alpha\left(f_{i}^{e q}-f_{i}\right)=0
$$

where $f_{i}^{e q}-f_{i}<0$, which guarantees positivity of the particle distribution in the node. The lower estimate $\alpha_{\min }>0$ can be computed from a rather complicated relation defined in [18]. In the developed simulation the Newton method is employed where the upper estimate $\alpha_{\max }$ plays the role of the starting value as it is efficient and preserves stability. If the calculation of the $\alpha$ does not converge to the solution of Eq. (2) then it is assumed that the value $\alpha_{\max }$ takes the role of the parameter $\alpha$. To eliminate computational demands the parameter $\alpha$ is evaluated only in nodes exceeding a tolerance value for the deviation $\left|\left(f_{i}^{e q}-f_{i}\right) / f_{i}\right|<10^{-2}$, i.e., in nodes with large deviations of the population $f_{i}$ from local equilibrium $f_{i}^{e q}$.

The computational domain is covered by fixed rectangular lattice with 320 thousands of nodes and local operations over all nodes are parallelized. Thus parallelization of computations is employed especially in the cases of evaluations of distribution functions $f_{i}$ and calculations of their moments (density $\rho$ and velocity $u$ ).

Parallelization of computations is provided by employment of the CUDA GPU computing technology within the MATLAB environment (and the associated Parallel Computing Toolbox). It means that all field quantities are transferred to the GPUs where operations are performed on them. The MATLAB environment is chosen partly for easy implementation of mathematical functions and structures and partly for the possibility of collective applications of operations over all nodes.

The possibility of straightforward parallelization of the LBM simulations yields considerable advantage from the point of efficiency. On the other side, the fact that the lattice grid is fixed brings substantial increase in computational demands in the case of extensive refinement. This problem can be resolved by employing adaptive meshes in regions with significant velocity gradients, e.g., [19].

\section{Results}

For each glass ball-mixture flow rate $(Q=0.58,0.66,0.76$, and $0.84 \mathrm{l} \mathrm{s}^{-1}$ ) one representative trajectory of an individual particle is chosen - from a number of observed trajectories, see in [1] - and compared to the path of the simulated particle. As just trajectories without mutual particle collisions were experimentally observed it can be assumed that motion of simulated particles consists mainly of free translational and rotational motion and collisions with the bed.

Simulation of particle motion is evaluated in a domain of the length $l=80 \mathrm{~mm}$ and height $h=40 \mathrm{~mm}$ which corresponds to the part of the optical box where the particle trajectories were observed. The measured velocity profiles are used for generation of the flow within the domain. Specifically, the profiles are induced at the inlet of the simulated region and let to develop above the rough bed. Into such a fully developed flows the particles are released from experimentally obtained initial positions $\left(x_{0}, y_{0}\right)$ with initial velocities $\left(v_{x 0}, v_{y 0}\right)$.

One experimental trajectory is chosen for each velocity profile and it is compared with the corresponding simulated trajectory. The couples of trajectories are drawn in Figs. 3 with respect to the length and height of the domain expressed in lattice space units. In the most upper figure the two trajectories - experimental and simulated - for the flow rate $Q=0.581 \mathrm{~s}^{-1}$ depart in the end of the examined domain. The second couple of trajectories for the flow rate $Q=0.661 \mathrm{~s}^{-1}$ varies in the location of the collision with the bed. In the third case, for the flow rate $Q=0.761 \mathrm{~s}^{-1}$, the particles do not collide with the bed and they are almost identical. Finally, the particles conveyed by the flow with $Q=0.841 \mathrm{~s}^{-1}$ diverge at the outflow of the observed domain.

It can be concluded that simulated trajectories differ partially from measured trajectories. There are more rea- 

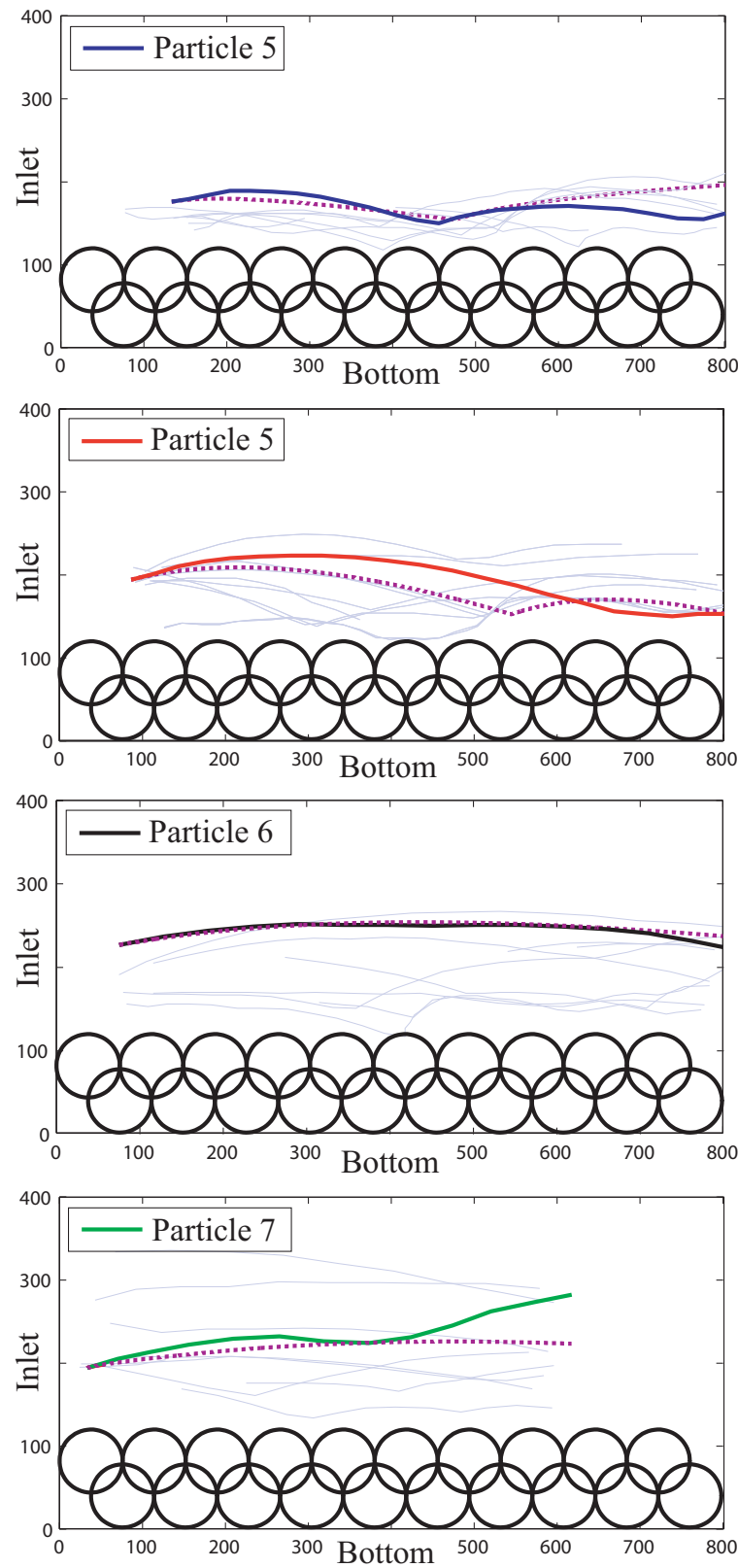

Fig. 3. Comparisons of experimental and simulated trajectories. The highlighted curves represent the compared trajectories. Specifically, the purple/dashed ones stand for the simulated trajectories while the experimental trajectories are drawn in color matching the velocity profiles in figure 2 . From top to bottom figures correspond to the flow rates in order $Q=0.581 \mathrm{~s}^{-1}, Q=0.66$ $1 \mathrm{~s}^{-1}, Q=0.761 \mathrm{~s}^{-1}$ and $Q=0.841 \mathrm{~s}^{-1}$. In the background other measured trajectories of particles with different initial values can be seen depicted as tiny curves of an opaque color.

sons which can cause these differences. First of all, the experimental particles are conveyed in the three-dimensional pipe while the developed simulation evaluates trajectories in the two-dimensional domain. Thus possibility of comparison of experimental and simulated trajectories consists in assuming that transversal velocities of the particles are more or less insignificant. However, it is obvious that to determine particle trajectories more properly lateral motion must be taken into account and it is necessary to introduce the third dimension into the simulation.
Another cause of discrepancies can be the fact that arrangements of the stationary particles in the experiment are not quite well defined. Irregular layout pattern can be resolved by employment a random number generator used to pick out one location from all possible contact points [10]. However, from the shapes of some experimental trajectories it can be seen that there are missing stationary particles in the higher level of the bed. In contrast, in the simulation a fixed layout pattern and unchanging height of the bed (i.e., made of two layers) is assumed.

Another problem is represented by the way of release of the simulated particle into the flow. Even if the particle is placed in the corresponding initial position with the right initial velocity in the fully developed flow its insertion in the domain induces unphysical disturbances at least in the start of the motion. This problem can be partly eliminated by introducing periodic boundary conditions through which the particle can enter the domain repeatedly.

Finally, for illustration six snapshots of the instantaneous flow field are shown in figure 4. It represents one of visual outputs of the developed simulation and should demonstrate the capability of the LBM to simulate both the particle motion and the fluid flow.

\section{Conclusions}

The LBM based simulation is developed to model particle motion in the water flow in the closed horizontal conduit. The simulation evaluates both the particle motion and velocity field of the flow. As the process is characterized by a high Reynolds number (up to $10^{5}$ ) the entropic extensions of the LBM is employed to guarantee stability of computation. Enhancement of computational resources is reached by parallelization of computations using the CUDA GPU technology. Assessment of accuracy of the used numerical approach is achieved by comparison of outputs of experimental measurements with results of the developed simulation.

It is shown that the compared - experimental versus simulated - trajectories given by identical initial values are quite similar though there are differences which can be caused by various reasons. In the simulation section 5 and the result section 6 a number of problems is addressed. Each of them has to be solved to obtain an accurate, stable and efficient simulation.

Firstly, as the entropic extension of the LBM depends largely on the correctness of the calculated value $\alpha$ its calculation must be improved in such a way that it will be both sufficiently accurate and efficient. This can be reached by the modification of both the numerical method and its implementation.

For the second the simulation must be rewritten for three-dimensional domain as this influences the processes fundamentally though such a transformation yields increase of computational demands and additional implementation issues.

Finally, validation of accuracy of the simulation will require additional more detailed experimental data including, e.g., particle-particle collisions, three-dimensional pattern of the bed etc. Verifications against experimental data belong to necessary resources which determine following steps in development of the simulation. 

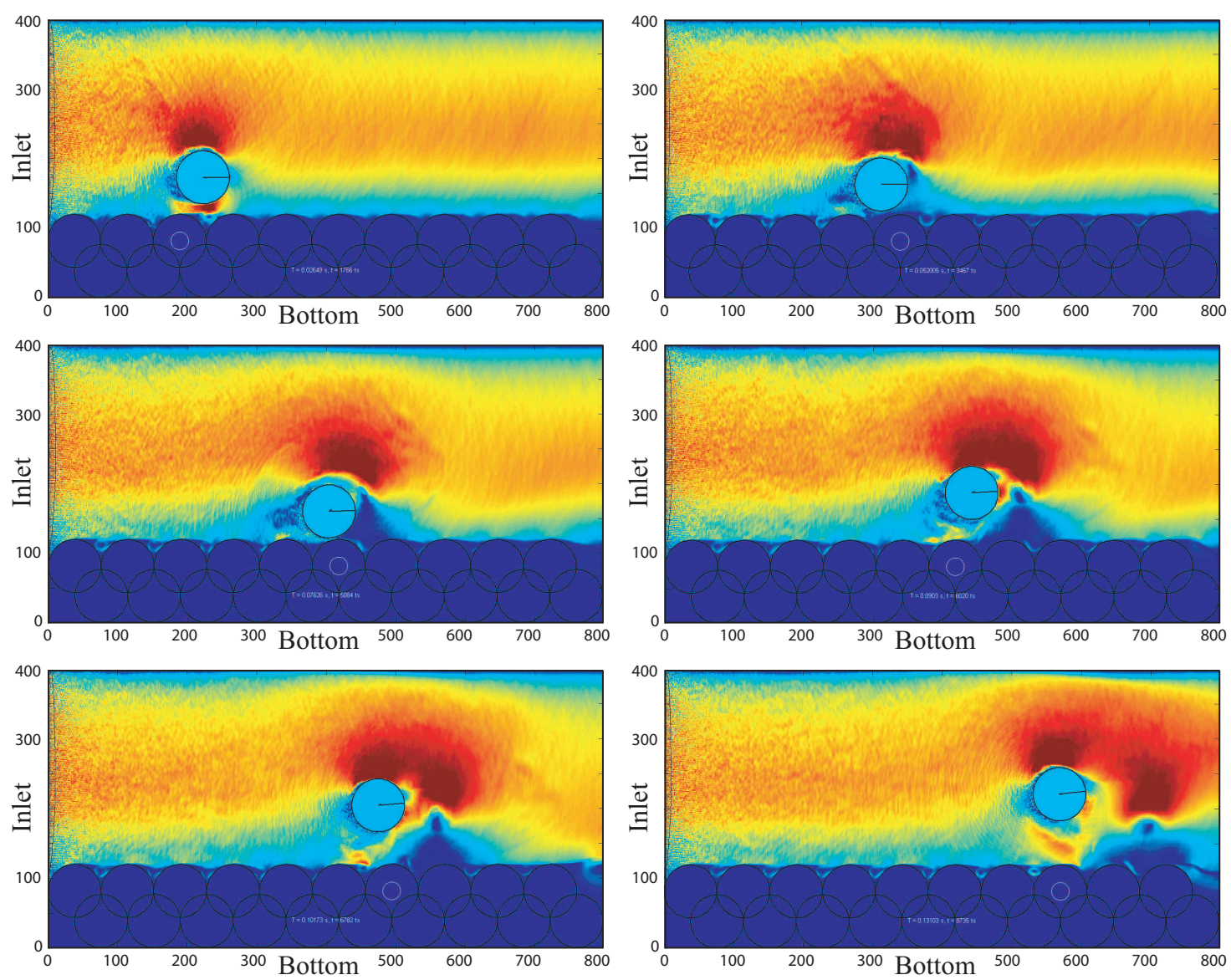

Fig. 4. Six snapshots of the instantaneous flow field are shown. They illustrate both the particle motion and the fluid flow as evaluated in a unique computational frame of the developed LBM based simulation. The first three figures depict conditions before a collision while the last three figures represent situations after the collision.

Further efficiency improvements in the algorithm and its parallelization will enable to simulate particle-fluid systems at the particle scale and thus can help to understand better the underlying particle-fluid dynamics. For example, in the considered process high level turbulence with large eddies along the bed influences the particle motion mode due to intensive mutual interactions of particles and the flow (and particle-wall collisions). Such a simulation tool could provide quantitative data for turbulence modeling difficult to obtain experimentally.

\section{Acknowledgements}

The support under the project No. P105/10/1574 of the GA CR and RVO: 67985874 of the Academy of Science of the Czech Republic is gratefully acknowledged.

\section{References}

1. P. Vlasák, B. Kysela, Z. Chára, Part. Sc. Tech. 32, (2013) 179-185

2. U. Frisch, B. Hasslacher, Y. Pomeau, Phys. Rev. Lett. 56, (1986) 1505-1508

3. G. R. McNamara, G. Zanetti, Phys. Rev Lett. 61, 1988 2332-2335
4. S. Succi, The lattice Boltzmann equation for fluid dynamics and beyond (Clarendon, Oxford, 2001)

5. X. He, L.-S. Luo, J. Stat. Phys. 88, (1997) 927-944

6. Z. Yu, L. S. Fan, Particuology 8, (2010) 539-543

7. P. Vlasák, B. Kysela, Z. Chára, J. Hydrol. Hydromech. 60, (2012) 115-124

8. P. L. Wiberg, J. D. Smith, J. Geophys. Res. 90 (1985) 7341-7354

9. W. Czernuszenko, Arch. Hydro-Eng. Env. Mech., 56 (2009) 101-120

10. N. Lukerchenko, Z. Chára, P. Vlasák, J. Hydraul. Res. 44, (2006) 70-78

11. N. Lukerchenko, N., Piatsevich, S., Chára, Z. and Vlasák, P., J. Hydrol. Hydromech. 57 (2009) 100-112

12. A. J. C. Ladd, J. Fluid Mech. 271, (1994) 311-339

13. Q. Zou, X. He, Phys. Fluids 9, (1997) 1591

14. C. K. Aidun, Y. Lu, E. J. Ding, J. Fluid. Mech. 373, (1998) 287-311

15. M.P. Allen, D. J. Tildesley, Computer Simulation of Liquids (Clarendon, Oxford, 1987)

16. S. Ansumali, I. V. Karlin, J. Stat. Phys. 107, (2002) 291-308

17. S. S. Chikatamarla, S. Ansumali, I. V. Karlin, Phys. Rev. Lett. 97, (2006) 010201

18. A. N. Gorban, I. V. Karlin, V. B. Zmievskii, T. F. Nonnenmacher, Physica A 231, (1996) 648-672

19. Z. Yu, L. S. Fan, J. Comp. Phys. 228, (2009) 64566478 